\title{
Frontières
}

\section{La musicothérapie en fin de vie Une voie de communication et d'apaisement}

\section{Nathalie Leduc}

Volume 20, numéro 2, printemps 2008

Les musiques et la mort

URI : https://id.erudit.org/iderudit/018352ar

DOI : https://doi.org/10.7202/018352ar

Aller au sommaire du numéro

\section{Éditeur(s)}

Université du Québec à Montréal

ISSN

1180-3479 (imprimé)

1916-0976 (numérique)

Découvrir la revue

Citer cet article

Leduc, N. (2008). La musicothérapie en fin de vie Une voie de communication et d'apaisement. Frontières, 20(2), 83-86. https://doi.org/10.7202/018352ar
Résumé de l'article

Cet article traite de la musicothérapie en soins palliatifs. La perspective d'une mort prochaine change considérablement la vie du malade et de sa famille, ce qui engendre souvent des difficultés à communiquer. Nous verrons comment la musicothérapie aide cette clientèle lorsque la parole ne suffit plus. La musique facilite l'expression des émotions et la communication avec le malade et sa famille afin de les apaiser. Nous approfondirons le pouvoir thérapeutique de la musique dans ses aspects expressifs, communicatifs, symboliques, de réminiscence, transitionnels et créatifs. Le processus thérapeutique sera décrit à partir d'histoires de cas d'enfants et d'adultes en fin de vie. Ces vignettes montreront comment la musicothérapie leur a permis d'exprimer des émotions en lien avec la fin de vie (colère, tristesse...), de maîtriser leur angoisse face à la mort ainsi que de profiter de moments de qualité avec leur famille. 


\section{Résumé}

Cet article traite de la musicothérapie en soins palliatifs. La perspective d'une mort prochaine change considérablement la vie du malade et de sa famille, ce qui engendre souvent des difficultés à communiquer. Nous verrons comment la musicothérapie aide cette clientèle lorsque la parole ne suffit plus. La musique facilite l'expression des émotions et la communication avec le malade et sa famille afin de les apaiser. Nous approfondirons le pouvoir thérapeutique de la musique dans ses aspects expressifs, communicatifs, symboliques, de réminiscence, transitionnels et créatifs. Le processus thérapeutique sera décrit à partir d'histoires de cas d'enfants et d'adultes en fin de vie. Ces vignettes montreront comment la musicothérapie leur a permis d'exprimer des émotions en lien avec la fin de vie (colère, tristesse...), de maîtriser leur angoisse face à la mort ainsi que de profiter de moments de qualité avec leur famille.

Mots clés: musicothérapie - musique - mort - expression - réminiscence symbolique - transitionnel.

\begin{abstract}
This article deals with music therapy in palliative care. The prospect of death changes enormously the life of the patient and his/her family, this often leads to difficulties in communicating. We will see how music therapy comes to the help of these clients when speech is not enough anymore. Music facilitates the expression of emotions and communication with the patient and his family, bringing them some appeasement. We will look in depth at the therapeutic power of music in its expressive, communicative, symbolic, reminiscent, transitional and creative aspects. The therapeutic process will be described using stories both from children's and adult's cases toward the end of their life. These " vignettes " will show how music therapy allows expression of emotions related with end of life (anger, sadness...), overcoming fear of dying, and sharing quality moments with family.

Keywords: music therapy - music - death - expression - reminiscent - symbolic transitional.
\end{abstract}

\section{La musicothérapie en fin de vie Une voie de communication et d'apaisement}

\section{Nathalie Leduc, M. A., musicothérapeute, département d'hémato-oncologie du CHU Sainte-Justine, Montréal.}

La maladie terminale affecte la vie d'un individu de manière significative. Les changements importants qui surviennent, qu'ils soient physiques, cognitifs, émotifs ou relationnels, peuvent empêcher la personne malade de s'exprimer et de communiquer aisément avec ses proches. Que faire lorsque la parole ne suffit plus? La musicothérapie peut venir en aide aux personnes en fin de vie en leur offrant une voie d'expression et de communication qui se situe au-delà de la parole.

Dans cet article, nous définirons la musicothérapie en soins palliatifs. Nous approfondirons le pouvoir thérapeutique de la musique dans ses aspects expressifs, communicatifs, symboliques, de réminiscence, transitionnels et créatifs. Nous décrirons le processus thérapeutique sousjacent à l'aide de vignettes où des enfants et des adultes se sont révélés dans toute leur simplicité, authenticité et créativité.

\section{LA MUSICOTHÉRAPIE EN SOINS PALLIATIFS}

Ayant travaillé comme musicothérapeute en soins palliatifs avec des adultes dans divers hôpitaux montréalais, j’ai eu la chance de faire partie d'équipes multidisciplinaires dont la préoccupation première était de veiller au bien-être du malade dans sa globalité, tant sur les plans physique, cognitif que psychosocial. La musicothérapie, bien intégrée à ces équipes de soins, apporte une touche complémentaire qui permet de répondre aux besoins multidimensionnels de cette clientèle grâce au médium non verbal qu'est la musique.

L'utilisation judicieuse de la musique live, tout particulièrement, facilite le contact humain, l'expression des émotions et la communication avec le malade et sa famille en proposant un apaisement, un confort physique et un soutien émotionnel.

\section{ASPECTS EXPRESSIFS \\ ET COMMUNICATIFS}

La détérioration de la condition physique et la médication peuvent limiter le patient dans sa capacité à communiquer avec l'entourage, soit en raison d'un état de faiblesse extrême, d'une altération de la conscience ou d'un état de confusion. Malgré les difficultés de communication, le patient est sensible aux stimuli sensoriels qui l'entourent tels que l'arrivée d'une personne dans sa chambre, le toucher, les sons ainsi que les paroles environnantes. Le son rejoint l'être humain à un niveau très primaire. Pensons au fotus qui, dès 
l'âge embryonnaire de quatre mois, entend les battements du cœur et la voix de sa mère. L'ouïe, au même titre que le toucher, est l'un des premiers sens à nous habiter.

Lorsque je joue d'un instrument de musique ou que je chante au chevet d'un de ces patients, des réponses non verbales sont observées : une respiration plus audible, un clignement des yeux, un contact visuel et un changement dans l'expression $\mathrm{du}$ visage. Ces manifestations corporelles sont souvent très apaisantes pour les proches et elles leur permettent d'observer et de réaliser qu'il est encore possible de communiquer avec la personne.

Le caractère prévisible et familier de la musique sécurise le malade qui, devant la perspective de mourir, perd ses repères. Le patient ne peut demeurer constamment dans l'intensité de la peine; il a besoin de normalité, d'être en contact avec un point d'ancrage, de renouer avec le calme, la paix, la joie et le plaisir afin de retrouver un équilibre émotionnel. Lorsqu'un patient est particulièrement vulnérable, le médium de la musique lui permet d'exprimer indirectement ce qu'il n'oserait peut-être pas formuler ouvertement. Des patients m'ont dit à maintes reprises: "Cette chanson va me rendre heureux.» Une telle affirmation sous-entend que la personne a des raisons d'être triste. Je me rappelle d'une jeune femme qui m'a demandé de jouer pour elle l'Ave Maria de Gounod, mais en faisant précéder et suivre cette pièce musicale par une œuvre plus légère comme la Petite musique de nuit de Mozart. Le fait de structurer ainsi la séance de musicothérapie lui a permis de contenir sa peine tout en exerçant un peu de contrôle sur son environnement.

Sur le plan psychologique, l'expérience de fin de vie peut s'avérer souffrante et angoissante pour le patient et sa famille. Parfois, les mots ne suffisent pas pour traduire ce qui se vit; il peut aussi arriver que l'expression verbale soit trop chargée émotivement. L'aspect non verbal de la musique est ici un atout précieux qui peut ouvrir la porte à une façon non menaçante de se relier et de se révéler à l'autre en situation de crise. Selon Leduc (1999a), il est souvent plus facile de choisir et d'écouter une chanson triste avec sa famille que d'exprimer cette tristesse avec des mots. "There would be no music and no need for it if it was possible to communicate verbally which is easily communicable musically»(Gaston, 1968).

\section{ASPECTS SYMBOLIQUES}

La musique et ses aspects symboliques permettent à l'individu d'exprimer un vaste spectre d'émotions telles la tristesse, la colère, l'angoisse, la joie pour n'en

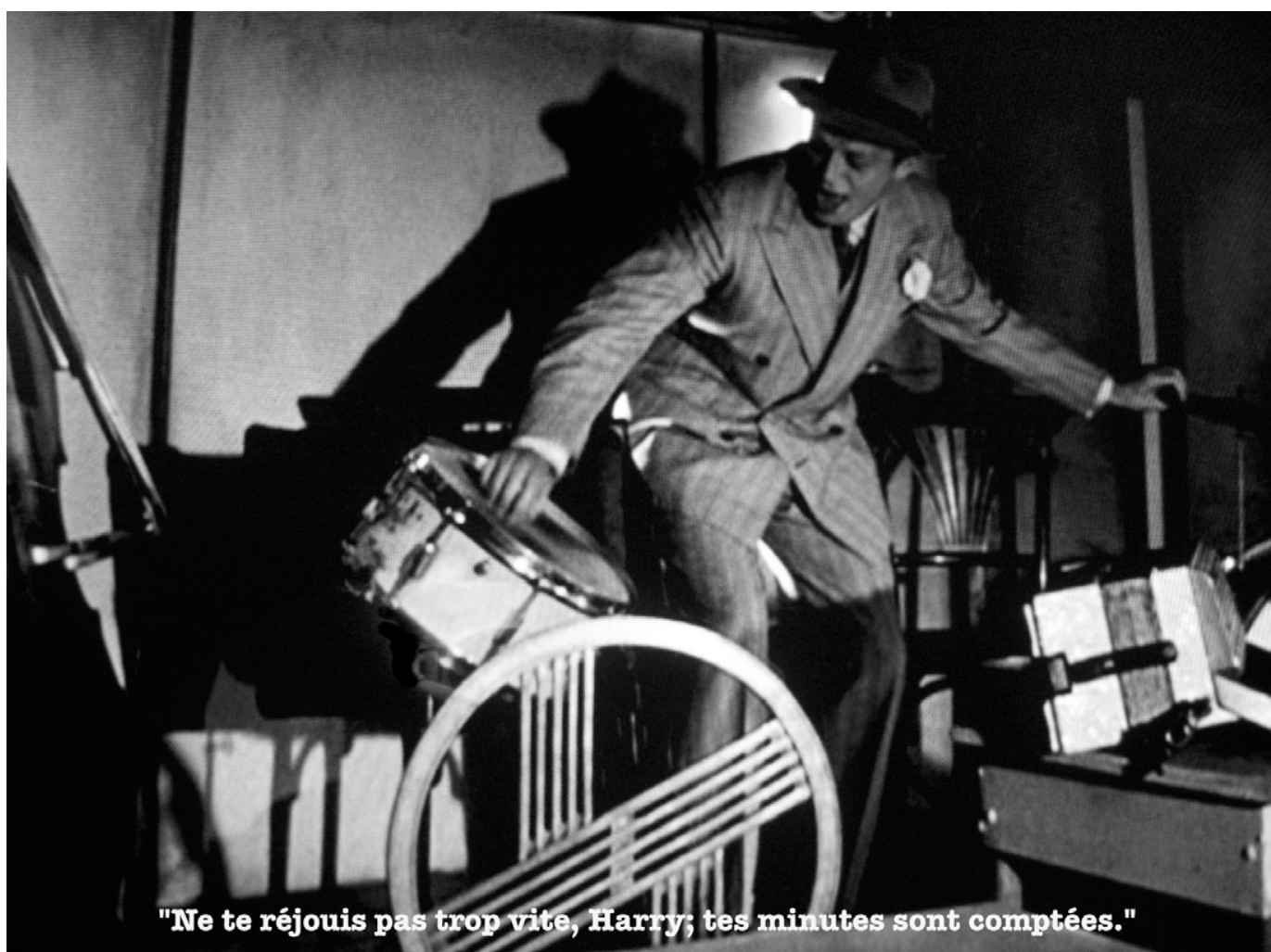

Louis CUMMINS, Film noir, « Harry », 2007, photographie tirée du film de Jules Dassin, Night in the City, $41 \mathrm{~cm} \times 65 \mathrm{~cm}$, collection de l'artiste.

nommer que quelques-unes. Une tonalité mineure suscitera souvent de la tristesse tandis qu'un rythme percutant pourrait plutôt traduire de la colère. Par l'intermédiaire de la musique, chaque individu peut s'engager dans une recherche de sens en lien avec son propre vécu. Une mélodie au violon pourra rendre nostalgique une personne d'origine italienne qui s'ennuie de son pays natal tandis qu'une autre dame se rappellera un moment heureux de son enfance lorsqu'une tante jouait de cet instrument à la maison. Les préférences musicales de la personne sont prises en compte pour la rejoindre dans ce qu'elle vit.

Selon Salmon (1995), la musique et les émotions partageraient un terrain commun qui réside dans l'interrelation entre les phénomènes de tension et de résolution. Elle mentionne que les composantes de la musique dans l'entrelacement et le contraste des dissonances et consonances, des tonalités mineures et majeures, des nuances douces et fortes, des tempos accélérés et ralentis font écho au vécu affectif de la personne en phase terminale. Dans cette perspective de tension et de résolution, le musicothérapeute adapte judicieusement les éléments musicaux afin de soutenir le patient dans l'expression des affects qui l'habitent tout en l'aidant à y trouver l'apaisement dont il a besoin. "When the emotional is too painful to bear, the musical framework may sup- port the patient providing holding, help to ventilate the overload content and more» (Sekeles, 1999, p. 178).

\section{ASPECTS DE RÉMINISCENCE}

Que ce soit sous forme d'images ou d'idées, la musique permet d'évoquer des souvenirs et les émotions qui y sont associées: "Terminally ill patients often engage in a life review process shifting through joy and disappointment and looking for pattern of meaning»(Salmon, 1993, p. 50). Je me rappelle d'un patient de 55 ans et de son épouse qui, en écoutant le son des vagues produit par un tambour océanique, se remémoraient avec tristesse comment le début de la maladie du conjoint les avait empêchés de partir en voyage aux Bermudes. Au contact de la musique, l'homme disait ressentir aussi du plaisir car, dans l'imaginaire, il pouvait faire ce voyage tant espéré. Il avait l'impression de reprendre le temps perdu. L'échange mutuel de souvenirs et l'expression des émotions qui en découlent grâce à la musique permet un rapprochement où patients et familles trouvent soulagement et apaisement dans la poursuite de leurs deuils respectifs.

\section{ASPECTS TRANSITIONNELS}

Lors de l'écoute d'une de nos chansons favorites, il n'est pas rare de ressentir du plaisir en se remémorant de bons souvenirs tout en étant nostalgique d'un temps passé 
qui n'est plus. La musique peut donc être à la fois évocatrice et reflet de la finitude. Elle permet ainsi le chevauchement entre deux réalités en y faisant osciller les enjeux qui entourent la vie et la mort, soit l'espoir de guérir versus la perspective de mourir. Winnicott (1953) considère l'écoute de la musique comme une expérience transitionnelle nourrissante qui facilite le passage entre deux réalités. Pensons simplement au fait de bercer un enfant en fredonnant pour qu'il s'endorme. Dans un contexte de soins palliatifs, la musicothérapie offre une expérience intermédiaire sécurisante qui contribue à aider le malade et sa famille à apprivoiser les derniers instants de vie. Souvent le patient résistera au sommeil par crainte de ne plus se réveiller. La relaxation que peut lui procurer un accompagnement par la musicothérapie favorise un lâcher prise à un niveau symbolique.

\section{ASPECTS CRÉATIFS}

La musicothérapie offre une variété de modalités prenant appui sur l'expression non verbale: les expressions du visage, les pieds qui battent la mesure sous les couvertures, les sessions d'improvisation musicale, l'écriture d'une chanson, la verbalisation en lien avec l'écoute d'une pièce de musique, etc. La musicothérapie fait donc appel à la créativité de la personne, soit la partie saine de son être, là où elle pourra trouver la force d'envisager les enjeux qui entourent sa maladie et peutêtre entrevoir une manière constructive de les gérer. De cette manière, la personne renoue avec ses ressources intérieures, ce qui pourra l'aider à mieux s'adapter à son environnement (voir Leduc, 1999b) et à conserver une certaine forme de dignité dans les derniers instants de sa vie. Encourager l'émergence d'un processus créatif peut aussi être thérapeutique pour la famille. Lorsqu'une maman chante pour son bébé mourant, elle renoue avec son rôle de mère malgré l'hospitalisation et la maladie. Elle peut ainsi oublier un instant son sentiment d'impuissance. Le chant de la mère est un élément naturel qui l'unit à son enfant (Nolan, 2003). Ces moments d'attachement de qualité restent imprégnés dans la mémoire des proches et facilitent leur deuil ultérieur.

\section{HISTOIRES DE CAS}

Les vignettes qui suivent ont été rédigées à partir de mon expérience clinique auprès de jeunes ( 9 et 13 ans) et d'adultes atteints de diverses formes de cancer, durant l'année qui a précédé leur décès.

\section{J'ENTENDS SON VIOLON}

Madame B., 84 ans, est en phase terminale d'un cancer du sein. Ayant accompagné son mari deux années plus tôt, elle affirme tout savoir sur les soins palliatifs et entre à l'hôpital bien déterminée à y attendre la mort. Elle ne se doute pas qu'elle a encore une multitude de choses à vivre, à exprimer et à partager, ne serait-ce qu'en profitant de chaque moment.

Madame B. affectionne tout particulièrement la chanson de Michèle Richard: J'entends son violon.

J'entends son violon qui joue des airs d'autrefois pour moi

J'entends son violon partout et j'entends sa voix

Il me dit ne pleure pas, je suis là tout près de toi

Et quand tu souris, moi je souris aussi

Lorsque la musicothérapeute joue cette chanson, tout l'être de Madame B. s'anime et s'illumine. Elle lui raconte des bribes

\section{LE CARACTÈRE PRÉVISIBLE}

\section{ET FAMILIER DE LA MUSIQUE}

SÉCURISE LE MALADE QUI,

DEVANT LA PERSPECTIVE

\section{DE MOURIR, PERD SES REPÈRES.}

d'un passé heureux où, ayant travaillé avec son époux dans l'alimentation, elle a maintes fois rencontré la chanteuse. Madame B. verbalise sa tristesse. Le contexte d'écriture de cette chanson (écrite en mémoire du père de l'interprète, «Ti-Blanc» Richard) et la nostalgie qui se dégage des paroles et de la musique ont permis à Madame B. de s'identifier au deuil de la chanteuse pour exprimer sa propre peine d'avoir perdu son époux ainsi que d'avoir à quitter ses proches qui lui sont chers. Avec beaucoup d'émotion, elle ajoute les paroles suivantes à la chanson: "J'entends ton violon qui me dit que tout n'est pas fini.»

Ce vécu, empreint d'une grande sensibilité, elle accepte ensuite de le partager à l'occasion d'un reportage télévisé sur la musicothérapie en soins palliatifs. Au-delà de la dame dont la mémoire n'est plus très fidèle et qui perd graduellement son autonomie physique, elle révèle à sa famille et au personnel soignant une image saine d'elle-même, jusqu'ici insoupçonnée: celle d'une femme sensible et expressive encore capable de prendre des décisions et d'avoir des projets qui lui sont propres comme la participation à un reportage télévisé. $\mathrm{Ce}$ processus créatif a permis à Madame B. de poursuivre ses deuils et de renouer avec des ressources intérieures lui permettant de s'épanouir davantage en dépit de la maladie et d'une mort prochaine.

\section{COU COU}

D., 9 ans, est en rechute d'un ostéosarcome (tumeur de l'os) qui ne peut être traité davantage. Elle est suivie en séances individuelles de musicothérapie dans une clinique externe depuis un an. Consciente qu'elle est en train de perdre la bataille, un matin, elle arrive en musicothérapie dans un état d'abattement. Sans adresser un mot ni un regard à la musicothérapeute, elle s'assoit et croise ses bras sur la table pour y poser sa tête. L'intervenante cesse de jouer de la guitare pour un moment et attend. Tout à coup, de la main droite, D. effleure $\mathrm{du}$ bout des doigts un xylophone pour y faire entendre des petits sons doux, aigus, à peine audibles. La musicothérapeute l'imite tout doucement à la guitare. Un dialogue musical s'installe. Par la suite, D. fait glisser de droite à gauche une baguette sur la table en émettant une succession de sons sourds. L'intervenante suit l'enfant dans son jeu en frappant le bout de ses ongles sur la guitare, dans une pulsation régulière, pour l'aider à s'organiser et subséquemment elle y incorpore des rythmes pour la stimuler. Au bout d'un moment, D. relève sa tête et s'engage davantage dans l'exploration de divers instruments de percussion qu'elle manipule avec véhémence. Un jeu rythmique énergique et bien organisé se développe. Finalement, D. initie un jeu de cache-cache à l'aide d'un gros tambour où, à tour de rôle, la musicothérapeute et l'enfant prennent place derrière l'instrument pour s'y cacher.

Au-delà des mots, le médium de la musique a rejoint $\mathrm{D}$. dans sa détresse en lui donnant une voie d'expression suffisamment sécurisante pour qu'elle puisse s'ouvrir au monde extérieur. La musicothérapeute, par le biais de l'imitation, a tenté de faire écho au vécu affectif de l'enfant afin qu'elle soit entendue dans sa tristesse. L'ajout d'une pulsation régulière et de nouveaux motifs rythmiques à l'improvisation musicale aura aidé $\mathrm{D}$. à organiser sa tristesse et ainsi à lui donner un sens afin qu'elle puisse faire place à sa colère. Le jeu de cache-cache lui a permis de projeter ses angoisses de mort et de tenter de les maîtriser à un niveau symbolique. Cette activité coïncidait également avec la fin de la séance, soit le moment où nous devions nous séparer.

\section{GARDE-MOI PRÈS DE TON C CEUR}

Proche parente de l'auteure, Madame A, atteinte d'un cancer lui dit un jour: "Composons une chanson (paroles et 
musique) pour les enfants avec lesquels tu travailles, à l'Hôpital Sainte-Justine. »

Oh! Garde-moi près de ton cœur

J'ai tant besoin d'un peu plus de chaleur

Oh! Garde-moi près de ton cœur

Je partirai oubliant mes douleurs

Petit enfant aux pas tremblants

Ce n'est pas vivre ce qui m'arrive

Oh! Garde-moi près de ton cœur

J'ai tant besoin d'un peu plus de chaleur

Oh! Garde-moi près de ton cœur

Je dormirai, oubliant mes douleurs

Petit enfant aux pas tremblants

Je veux guérir car je veux vivre

Dans les premières paroles: "Gardemoi près de ton cœur», on peut lire un besoin de rapprochement ainsi qu'un besoin de tendresse: "J'ai tant besoin d'un peu plus de chaleur». Par l'emploi du pronom «je», Madame A. semble s'identifier aux enfants malades. Dans le contenu des paroles, l'aspect transitionnel est présent dans l'entrelacement vie/mort, le chevauchement de deux réalités : la guérison et la perspective du mourir. Ainsi, les paroles: "Je veux guérir car je veux vivre» et «Je dormirai, oubliant mes douleurs » sont en lien avec la guérison alors que les phrases: «Ce n'est pas vivre ce qui m'arrive » et «Je partirai oubliant mes douleurs» sont en lien avec la perspective de mourir.

Madame A. manipulait la structure de la chanson en lien avec le contenu des paroles. Elle semblait trouver plus sécurisant et moins confrontant de terminer la chanson avec la perspective de guérir plutôt qu'avec celle de mourir. Quand elle dit: «Ce n'est pas vivre ce qui m'arrive», elle ajoute: "c'est trop triste, finissons autrement par: " je vais guérir car..." ". Dans la composition de la musique, Madame A. a su utiliser certains éléments musicaux qui soutiennent le message exprimé par les paroles de la chanson. Ainsi, l'emploi d'une ligne mélodique ascendante accompagnée d'un crescendo lui a permis une ouverture pour l'expression de la perspective de mourir: "Ce n'est pas vivre ce qui m'arrive».

\section{QUAND LA MORT NOUS PÈSE}

E., 13 ans, est atteint d'une tumeur à son dernier stade. Il est vu par une musicothérapeute deux semaines avant son décès. En raison de la progression de sa maladie, le jeune est limité sur le plan verbal et physique. Il peut prononcer un mot à la fois et soulever légèrement son bras droit, mais le reste de son corps est paralysé.
Lorsque la musicothérapeute entre dans la chambre, un silence lourd se fait sentir. Le jeune ainsi que ses deux parents écoutent la télé pour passer le temps. L'intervenante s'approche du jeune en faisant entendre une petite harpe portative à sept cordes. Deux petits yeux expressifs et un sourire illuminent le visage de E., ému, et les parents s'approchent du lit pour partager avec lui son plaisir immédiat. En voyant que la mère manifeste de l'intérêt et de la curiosité pour la petite harpe, la musicothérapeute dépose l'instrument dans ses mains et l'encourage à explorer les sons. En voyant et en entendant sa mère jouer de la petite harpe, E. se met à rire. Il tend sa main voulant lui aussi prendre part au jeu. La mère s'approche de son enfant, lui prend la main et l'aide à jouer de la harpe.

À la fois rejoints par la musique et témoins du plaisir ressenti par leur enfant dans les derniers instants, les parents ressentent une vive émotion où joie et peine se chevauchent. La musique relie ici les membres d'une même famille et leur donne accès à un espace relationnel de grande qualité. C'est souvent dans l'intensité du lien que s'inscrit la précarité de la vie. La musicothérapie leur a permis de profiter du moment présent et ainsi d'avancer dans le temps afin de contrer une attente trop lourde, celle de la mort.

La musique est universelle: elle rejoint tout le monde quel que soit l'âge, le sexe, la culture et le statut (malade, famille, personnel soignant). La musicothérapie, dans l'entrelacement des sons et de la parole, ouvre de nouvelles voies d'expression et de communication afin de répondre aux besoins d'enfants et d'adultes en processus de deuil. Madame B, par l'écoute de sa chanson favorite, a verbalisé de bons souvenirs tout en exprimant la peine d'avoir à quitter ses proches. Grâce au reportage télévisé, les enfants de Madame B. garderont en souvenir des images réconfortantes de leur mère. D., par le biais de l'improvisation musicale, a traduit avec des sons sa tristesse et sa colère d'être en rechute. Madame A., en composant la musique et les paroles d'une chanson a pu confronter les enjeux vie / mort. La mère de E., en s'engageant musicalement avec son enfant, a permis à toute la famille de vivre un moment intense et de grande qualité. Le processus créatif, soutenu par la musicothérapie, a permis à ces personnes de s'actualiser dans le moment présent afin de diminuer leur sentiment d'impuissance tout en tentant de maîtriser leur angoisse de mort.

\section{Bibliographie}

ASSOCIATION DE MUSICOTHÉRAPIE DU CANADA (2008). Site Internet, <http:// www.musictherapy.ca/fr/index.htm>.

ASSOCIATION QUÉBÉCOISE DE MUSICOTHÉRAPIE (2008). Site Internet, <http:// www.musicotherapieaqm.com/>.

GASTON, E.T. (1968). Music in Therapy, New York, Macmillan.

LEDUC, N. (1999a). "Au-delà des mots, un son: La musicothérapie auprès des patients atteints de cancer ", Hope \& Cope Newsletter, p. 5-6.

LEDUC, N. (1999b). A Model of Supportive Music Therapy with Medically Ill Patients, thèse de maîtrise non publiée, MCP Hahnemann University, Philadelphie.

NOLAN, P. (2003). "Music and neonates: Food for life-composing the future", dans J. LOEWY (dir.), Music Therapy in the Neonatal Intensive Care Unit, New York, Satchnote, $2^{\mathrm{e}}$ éd., p. 121-125.

SALMON, D. (1995). "Music and emotion in palliative care: Accessing inner resources", dans C.A. LEE (dir.), Lonely Waters, Oxford, Sobells Publications, p. 71-84.

SALMON, D. (1993). "Music and emotion in palliative care ", Journal of Palliative Care, vol. $9, n^{\circ} 4$, p. 48-52.

SEKELES, C. (1999). "Working through loss and mourning in music therapy ", dans T. WIGRAM et J. DE BACKER (dir). Clinical Applications of Music Therapy in Psychiatry, préface de J. PEUSKENS, Londres, Jessica Kingsey Publishers, p. 176-196.

WINNICOTT, D.W. (1953). «Transitional objects and transitional phenomena », The International Journal of Psychoanalysis, $\mathrm{n}^{\circ} 34$, p. 89-97. 